\title{
Evaluation of Drug-Drug Interaction Potential between Baloxavir Marboxil and Oseltamivir in Healthy Subjects
}

\author{
Nao Kawaguchi ${ }^{1} \cdot$ Hiroki Koshimichi $^{1} \cdot$ Toru Ishibashi $^{1} \cdot$ Toshihiro Wajima $^{1}$
}

Published online: 10 September 2018

(c) The Author(s) 2018

\begin{abstract}
Background and Objective Baloxavir marboxil is a prodrug that is metabolized to baloxavir acid, which suppresses viral replication by inhibiting cap-dependent endonuclease with a single oral administration. As the mode of action of baloxavir marboxil is different from that of neuraminidase inhibitors, such as oseltamivir, combination treatment with these drugs can be a treatment option, particularly for severe influenza infection. The aim of this study was to assess the drug-drug interaction between baloxavir marboxil and oseltamivir.

Methods Eighteen healthy adult subjects received three treatments in a crossover fashion: single administration of baloxavir marboxil $40 \mathrm{mg}$ alone, repeated twice-daily administration of oseltamivir at $75 \mathrm{mg}$ for 5 days, or single administration of baloxavir marboxil at $40 \mathrm{mg}$ in combination with repeated twice-daily administration of oseltamivir at $75 \mathrm{mg}$ for 5 days.

Results The ratios (90\% confidence intervals) of maximum plasma concentration and area under the plasma concentration-time curve of baloxavir acid after co-administration compared to baloxavir marboxil alone were $1.03(0.92-1.15)$ and 1.01 (0.96-1.06), respectively. The ratios (90\% confidence intervals) of maximum plasma concentration and area under the plasma concentration-time curve of oseltamivir carboxylate, the active form of oseltamivir, after co-administration compared to oseltamivir alone were $0.96(0.93-1.00)$ and $0.99(0.96-1.01)$, respectively, at steady state on day 5 . Treatment-emergent adverse events reported were mild and not considered to be related to the study drug.

Conclusion The lack of a clinically meaningful drug-drug interaction between baloxavir marboxil and oseltamivir has been established.
\end{abstract}

\section{Key Points}

Baloxavir marboxil, a novel anti-influenza drug, and oseltamivir did not affect the mutual pharmacokinetics and no treatment-emergent adverse events related to the study drug were observed after combination treatment.

Combined with evidence from non-clinical studies, the combination treatment of baloxavir marboxil and oseltamivir has synergistic antiviral activity.

These findings support the use of combination treatment of baloxavir marboxil and oseltamivir without the need for dose adjustment.

Toru Ishibashi

toru.ishibashi@shionogi.co.jp

1 Clinical Pharmacology and Pharmacokinetics, Shionogi \& Co., Ltd., Umeda Office 12F, Hankyu Terminal Bldg., 1-4, Shibata 1-chome, Kita-ku, Osaka 530-0012, Japan

\section{Introduction}

Baloxavir marboxil (product code S-033188) has potent antiviral activity against influenza $A$ and $B$ viruses with a single oral administration. Baloxavir acid, the active form of baloxavir marboxil, selectively inhibits cap-dependent endonuclease, which is a key enzyme involved in the initiation of messenger RNA synthesis of influenza viruses; this is called a cap-snatching process [1]. Baloxavir marboxil suppresses viral replication by inhibiting the protein synthesis needed for the construction of new virions, which is a different mode of action from neuraminidase inhibitors such as oseltamivir (Tamiflu ${ }^{\circledR}$ ) widely used for the treatment of influenza. Combination treatment of baloxavir marboxil and oseltamivir shows synergistic antiviral activity in nonclinical studies $[2,3]$. Therefore, the combination treatment with these drugs can be a treatment option, particularly for seriously ill patients with influenza.

Baloxavir marboxil is a prodrug that is rapidly hydrolyzed to baloxavir acid by arylacetamide deacetylase in the small 
intestine, blood, and liver [4]. Baloxavir acid is primarily metabolized by uridine diphosphate glucuronosyl transferase $1 \mathrm{~A} 3$ with minor contribution from cytochrome P450 3A4 and approximately $80 \%$ of the administered drug is excreted via the feces [4]. Baloxavir marboxil is minimally detected in plasma, while baloxavir acid is mainly detected. Baloxavir acid exhibits linear pharmacokinetics over the dose range tested in a phase I study $(6-80 \mathrm{mg})$ and has a long elimination half-life, $80-100 \mathrm{~h}$ after a single oral administration [5]. Baloxavir marboxil and baloxavir acid have the potential to inhibit P-glycoprotein-mediated transport in an in vitro study [4].

Oseltamivir is orally administered with a twice-daily regimen for 5 days for the treatment of influenza [6]. Oseltamivir is also a prodrug and is hydrolyzed to the active form, oseltamivir carboxylate, by hepatic carboxylesterases. Oseltamivir carboxylate is excreted via the urine without further metabolism. Oseltamivir and oseltamivir carboxylate do not interact with substrates of various cytochrome P450 isoforms [7, 8]. Oseltamivir has been reported to be a substrate of P-glycoprotein [9] and peptide transporter 1 [10].

Drug-drug interaction (DDI) potential between baloxavir marboxil and oseltamivir is unclear and the DDI assessment can be valuable information for consideration of combination treatment. The aim of this study was to assess in vivo DDI potentials between baloxavir marboxil and oseltamivir on the basis of plasma concentration data of each prodrug and their active forms as well as a safety evaluation.

\section{Methods}

\subsection{Study Design}

This study was conducted in accordance with all appropriate regulatory requirements and under a protocol approved by the institutional review board of the study site, Souseikai Hakata Clinic, Japan. This study was conducted in accordance with current International Conference on Harmonisation Good Clinical Practice, all appropriate subject privacy requirements, and the ethical principles outlined in the Declaration of Helsinki. Informed consent was obtained from all subjects prior to the initiation of the study.

This study was a phase I, single-center, open-label, randomized, six-sequence, three-period, and three-treatment crossover study. The study was designed to assess the effect of oseltamivir on the pharmacokinetics and safety of baloxavir marboxil and its active form, baloxavir acid, as well as the effect of baloxavir marboxil on the pharmacokinetics and safety of oseltamivir and its active form, oseltamivir carboxylate. A total of 18 Japanese healthy adult male subjects aged from 20 to 59 years with a body weight of $\geq 50 \mathrm{~kg}$ and body mass index of $18.5-25.0 \mathrm{~kg} / \mathrm{m}^{2}$ were randomized to one of six sequence groups consisting of three subjects each. Key exclusion criteria were consumption of drugs including inducers of cytochrome $\mathrm{P} 450$ or uridine diphosphate glucuronosyl transferase enzymes or St. John's wort-containing products within 28 days prior to admission; consumption of any drug (prescription or over-the-counter drugs, dietary supplements, or vitamins) within 14 days prior to admission; use of alcohol- or grapefruit-containing products within $72 \mathrm{~h}$ prior to admission; and use of tobacco- or nicotine-containing products within 24 weeks prior to screening.

Each subject received all three treatments in a crossover fashion according to the randomized sequence. The treatments were as follows: single oral administration of baloxavir marboxil (Xofluza ${ }^{\mathrm{TM}}$ : Shionogi \& Co., Ltd. Osaka, Japan) at $40 \mathrm{mg}$ on day 1 in the fasted state; single oral administration of oseltamivir (Tamiflu ${ }^{\circledR}$ : F. Hoffmann-La Roche Ltd., Basel, Switzerland) at $75 \mathrm{mg}$ on day 1 in the fasted state, followed by repeated twice-daily administration of oseltamivir at $75 \mathrm{mg}$ until day 5 after each meal; co-administration of baloxavir marboxil at $40 \mathrm{mg}$ and oseltamivir at $75 \mathrm{mg}$ simultaneously on day 1 in the fasted state, followed by repeated twice-daily administration of oseltamivir at $75 \mathrm{mg}$ until day 5 after each meal. There was an at least 21-day washout interval between each treatment.

\subsection{Sample Collection}

Blood samples for the determination of plasma concentrations of baloxavir marboxil and baloxavir acid were collected at the following time points: pre-dose and 0.5, 1, 2, 3, 4, 5, $6,8,12,24,36,48,72,120,168,336$, and 504 h post-dose on day 1. Blood samples for the determination of plasma concentrations of oseltamivir and oseltamivir carboxylate were collected at the following time points: pre-dose and $0.5,1,2,3,4,5,6,8$, and $12 \mathrm{~h}$ post-dose on day 1 , pre-dose, and $12 \mathrm{~h}$ post-dose on day 4 , pre-dose and $0.5,1,2,3,4,5$, 6,8 , and $12 \mathrm{~h}$ post-dose on day 5 .

\subsection{Bioanalytical Asssay}

The bioanalytical methods for the determination of plasma concentrations of baloxavir marboxil and baloxavir acid were validated where the lower limit of quantification was set at $0.100 \mathrm{ng} / \mathrm{mL}$. Dichlorvos, as an inhibitor of esterases that metabolize baloxavir marboxil to baloxavir acid, was added to the plasma samples. Plasma samples were analyzed after deproteinization by the liquid chromatography tandem mass spectrometry method. The assay was linear from 0.100 to $300 \mathrm{ng} / \mathrm{mL}$. The precision levels of the assay were $2.8-7.4 \%$ and $2.4-6.1 \%$ for baloxavir marboxil and baloxavir acid, respectively. The accuracy levels of the assay were $2.0-6.0 \%$ and $-1.5 \%$ to $6.8 \%$ for baloxavir marboxil and baloxavir acid, respectively. 
The bioanalytical method for the determination of plasma concentrations of oseltamivir and oseltamivir carboxylate was validated with the lower limit of quantification set at 1.00 and $10.0 \mathrm{ng} / \mathrm{mL}$ for oseltamivir and oseltamivir carboxylate, respectively. Plasma samples were analyzed after deproteinization by the liquid chromatography tandem mass spectrometry method. The assay was linear from 1.00 to $500 \mathrm{ng} / \mathrm{mL}$ and from 10.0 to $5000 \mathrm{ng} / \mathrm{mL}$ for oseltamivir and oseltamivir carboxylate, respectively. The precision levels of the assay were $7.4-10.4 \%$ and $5.1-7.7 \%$ for oseltamivir and oseltamivir carboxylate, respectively. The accuracy levels of the assay were $-7.8 \%$ to $1.5 \%$ and $-2.1 \%$ to $4.2 \%$ for oseltamivir and oseltamivir carboxylate, respectively.

\subsection{Pharmacokinetic Analysis}

For the summary of plasma concentrations, plasma concentration below the limit of quantification will be treated as zero for calculations of arithmetic mean and standard deviation. For the calculation of pharmacokinetic parameters, plasma concentration below the limit of quantification before the occurrence of the first measurable concentration will be treated as zero, and plasma concentration below the limit of quantification after the first occurrence of the measurable concentration will be treated as missing.

The following pharmacokinetic parameters were calculated on the basis of plasma concentration data by noncompartmental methods using Phoenix WinNonlin software (Version 6.2.1; Certara USA, Inc., Princeton, NJ, USA): maximum plasma concentration $\left(C_{\max }\right)$, time to $C_{\max }$ $\left(T_{\max }\right)$, area under the plasma concentration-time curve from time zero to the time of the last quantifiable concentration after dosing $\left(\mathrm{AUC}_{0-\text { last }}\right)$, area under the plasma concentration-time curve extrapolated from time zero to infinity (AUC 0 -inf), and area under the plasma concentration-time curve over the dosing interval $\tau$, which is $12 \mathrm{~h}$ for administration of oseltamivir $\left(\mathrm{AUC}_{0-\tau}\right)$. Maximum plasma concentration, $T_{\max }, \mathrm{AUC}_{0-\text { last }}$, and $\mathrm{AUC}_{0 \text {-inf }}$ were calculated as pharmacokinetic parameters for baloxavir acid and $C_{\max }, T_{\max }$, and $\mathrm{AUC}_{0-\tau}$ were calculated as pharmacokinetic parameters for oseltamivir and oseltamivir carboxylate.

\subsection{Statistical Analysis}

Analysis of variance was performed using SAS (Version 9.2; SAS Institute Inc., Cary, NC, USA) for natural logarithmic-transformed values of $C_{\max }$ and AUC, which included factors of treatment, group, and period as fixed effects and subject as a random effect. In the case of unbalanced data, the Kenward-Roger method was used to compute the denominator degrees of freedom for the tests of a fixed effect in the analysis. The point estimates and $90 \%$ confidence intervals (CIs) were generated for the differences of logarithmic-transformed pharmacokinetic parameters between dosing alone and co-administration. The point estimates and the $90 \%$ CIs were back-transformed to obtain the corresponding geometric least-squares mean ratios (GMRs) and the $90 \%$ CIs.

\subsection{Safety Assessment}

Safety was assessed through physical examination, vital sign measurements, clinical laboratory tests, 12-lead electrocardiograms, and assessment of adverse events. Physical examination and vital sign measurement were performed at screening, on the admission day (only physical examination), pre-dose, 2, 4, 8, 12, 24, 48, 72, 96, and $120 \mathrm{~h}$ after the first dose, and on day 15 in each treatment, and at the end of the study or early termination examination. Clinical laboratory tests was performed at screening, on the admission day of period 1 , pre-dose, 24 , and $120 \mathrm{~h}$ after the first dose, and on day 15 in each treatment, and at the end of the study or early termination examination. The 12-lead electrocardiogram measurement was performed at screening, on the admission day, pre-dose, 1, 4, 24, and $120 \mathrm{~h}$ after the first dose in each treatment, and at the end of the study or early termination examination.

Adverse events were collected from the first dose through the end of the study examination. All safety data collected were assessed for severity and relationship to each study drug, baloxavir marboxil or oseltamivir. The severity of an event was graded as follows: mild: a finding or symptom was minor and did not interfere with usual daily activities; moderate: the event was discomfort and caused interference with the usual daily activity of affected clinical status; or severe: the event caused interruption of the subject's usual daily activities or had a clinically significant effect.

\section{Results}

\subsection{Study Subjects}

Eighteen Japanese healthy adult subjects were randomized into one of six sequence groups and received all three treatments (baloxavir marboxil alone, oseltamivir alone, and coadministration of these drugs). The mean (range) of age, body weight, and body mass index in the study population were $37.7(21-49)$ years, $63.4(53.7-76.7) \mathrm{kg}$, and 22.0 $(19.5-24.3) \mathrm{kg} / \mathrm{m}^{2}$, respectively.

One subject withdrew from the study on day 1 in period 1 after co-administration of baloxavir marboxil and oseltamivir. The subject's plasma sample was collected at up to $8 \mathrm{~h}$ (planned) and $10.27 \mathrm{~h}$ post-dose for early termination examinations. The plasma concentration at early termination was used for plotting as the concentration at $12 \mathrm{~h}$ post-dose. The 
subject's $\mathrm{AUC}_{0 \text {-last }}$ and $\mathrm{AUC}_{0 \text {-inf }}$ of baloxavir acid were not calculated because of a lack of concentration data in the terminal phase. The subject's pharmacokinetic parameters of oseltamivir and oseltamivir carboxylate on day 1 were calculated and included in the statistical analysis because the sampling time at early termination was close to the last sampling time of oseltamivir and oseltamivir carboxylate on day 1 ( $12 \mathrm{~h}$ post-dose), suggesting that the pharmacokinetic parameters could be calculated appropriately.

\subsection{Effect on Pharmacokinetics of Baloxavir Marboxil and Baloxavir Acid}

The plasma concentrations of baloxavir marboxil were below the limit of quantification at most of the sampling points when baloxavir marboxil was orally administered alone or in combination with oseltamivir. Their highest values for each treatment were $0.276 \mathrm{ng} / \mathrm{mL}$ at $8 \mathrm{~h}$ post-dose and $0.198 \mathrm{ng} /$ $\mathrm{mL}$ at $6 \mathrm{~h}$ post-dose. The calculation of pharmacokinetic parameters of baloxavir marboxil and the statistical analysis were not performed.

For baloxavir acid, the plasma concentration profiles when baloxavir marboxil was administered alone or in combination with oseltamivir were superimposable as shown in Fig. 1. The GMRs (90\% CIs) of $C_{\text {max }}, \mathrm{AUC}_{0 \text {-last }}$, and $\mathrm{AUC}$ 0 -inf of baloxavir acid after co-administration compared to baloxavir marboxil alone were 1.0288 (0.9190-1.1517), 1.0085 (0.9608-1.0585), and 1.0057 (0.9575-1.0563), respectively, as shown in Table 1 . The GMRs of $C_{\max }$ and AUC of baloxavir acid were close to 1 and the corresponding $90 \%$ CIs were contained within the range of $0.8000-1.2500$, which met no DDI effect criteria, [11-13] suggesting that

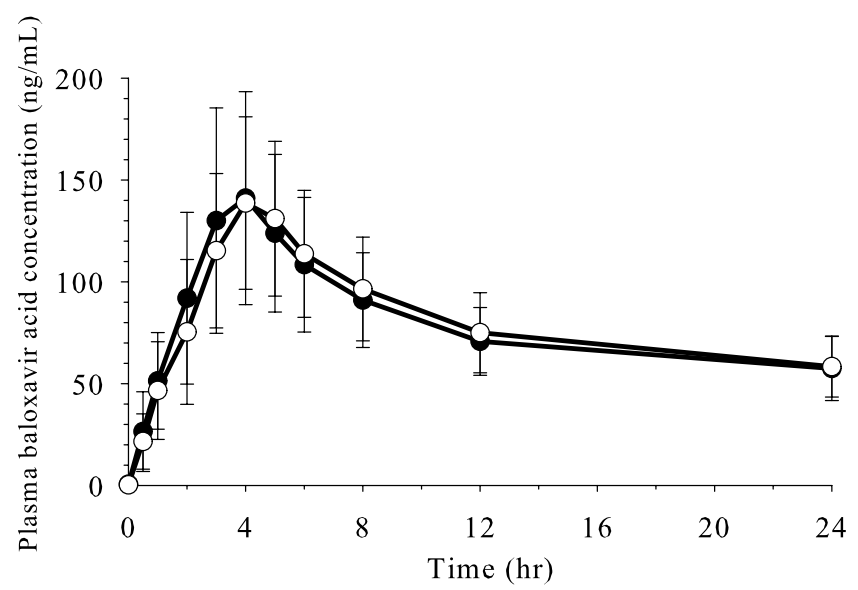

(a)

Fig. 1 Plasma concentration profiles of baloxavir acid when administered with or without oseltamivir. Mean ( \pm standard deviation) plasma concentration profiles with linear scale (a) [up to $24 \mathrm{~h}$ postdose] and semi-log scale (b). A closed circle indicates treatment with oseltamivir did not affect the pharmacokinetics of baloxavir acid.

\subsection{Effect on Pharmacokinetics of Oseltamivir and Oseltamivir Carboxylate}

The plasma concentration profiles of oseltamivir and oseltamivir carboxylate when oseltamivir was orally administered alone or in combination with baloxavir marboxil were superimposable after the first dose on day 1 and at steady state on day 5 as shown in Fig. 2.

$\mathrm{AUC}_{0-\tau}$ of oseltamivir on day 1 increased 1.23-fold after co-administration compared to oseltamivir alone as shown in Table 2. However, the GMRs ( $90 \%$ CIs) of $C_{\max }$ and AUC $0-\tau$ of oseltamivir on day 5 were $0.9559(0.8263-1.1058)$ and 1.0672 (0.9937-1.1462), respectively. From these results, the effect of baloxavir marboxil on the pharmacokinetics of oseltamivir was not remarkable. For oseltamivir carboxylate, the GMRs (90\% CIs) of $C_{\max }$ and $\mathrm{AUC}_{0-\tau}$ after coadministration compared to oseltamivir alone were 0.9594 (0.9074-1.0145) and $0.9591(0.9179-1.0021)$ on day 1 and $0.9648(0.9299-1.0010)$ and $0.9873(0.9630-1.0122)$ on day 5 , respectively, which met no DDI effect criteria. [11-13] Therefore, baloxavir marboxil did not affect the pharmacokinetics of oseltamivir carboxylate (Table 3).

\subsection{Safety}

Treatment-emergent adverse events were reported in six of 18 subjects. Treatment-emergent adverse events reported in two or more subjects were nausea and increased aspartate aminotransferase as shown in Table 4. Nausea was reported

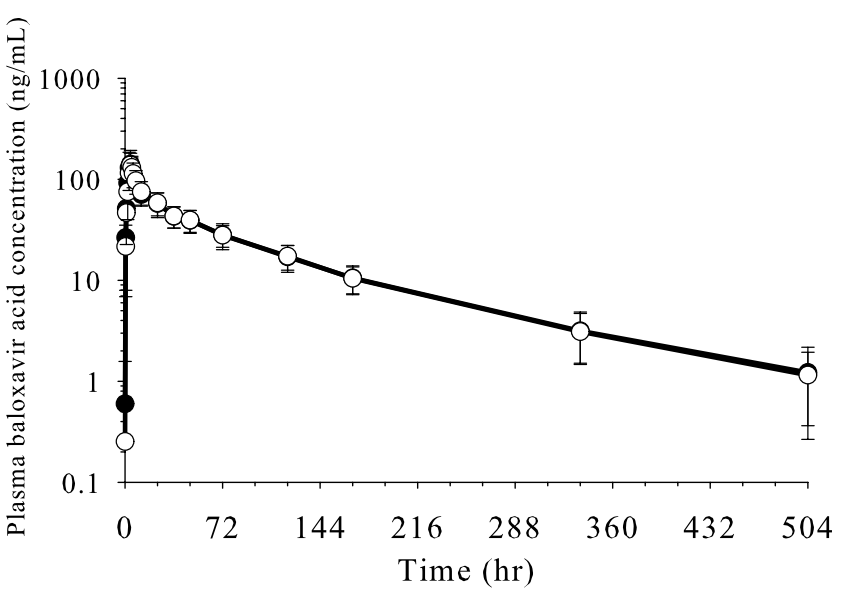

(b)

baloxavir marboxil alone $(n=17)$ and an open circle indicates treatment with co-administration of baloxavir marboxil and oseltamivir $(n=18)$ 
Table 1 Summary of pharmacokinetic parameters of baloxavir acid when administered with or without oseltamivir

\begin{tabular}{|c|c|c|c|}
\hline \multirow{2}{*}{ Parameter } & \multicolumn{2}{|l|}{ Geometric mean (CV\%) } & \multirow{2}{*}{$\begin{array}{l}\text { Geometric least-squares mean ratio }(90 \% \\
\text { confidence interval) }^{\mathrm{b}} \\
\text { Co-administration/baloxavir marboxil alone }\end{array}$} \\
\hline & $\begin{array}{l}\text { Baloxavir marboxil alone } \\
{[N=17]}\end{array}$ & Co-administration $[N=18]$ & \\
\hline$C_{\max }(\mathrm{ng} / \mathrm{mL})$ & $137(42.7)$ & $141(31.8)$ & $1.0288(0.9190-1.1517)$ \\
\hline $\operatorname{AUC}_{0-\text { last }}(\mathrm{ng} \cdot \mathrm{h} / \mathrm{mL})$ & $6574(29.5)$ & $6629(26.5)^{\mathrm{c}}$ & $1.0085(0.9608-1.0585)$ \\
\hline $\mathrm{AUC}_{0-\text { inf }}(\mathrm{ng} \cdot \mathrm{h} / \mathrm{mL})$ & $6764(30.5)$ & $6803(27.3)^{\mathrm{c}}$ & $1.0057(0.9575-1.0563)$ \\
\hline$T_{\max }{ }^{\mathrm{a}}(\mathrm{h})$ & $4.00(3.00-5.00)$ & $4.00(3.00-5.00)$ & - \\
\hline
\end{tabular}

$A U C_{0-i n f}$ area under the plasma concentration-time curve extrapolated from time zero to infinity, $A U C_{0-l a s t}$ area under the plasma concentrationtime curve from time zero to the time of the last quantifiable concentration after dosing, $C_{\max }$ maximum plasma concentration, $C V \%$ coefficient of variation, $T_{\max }$ time to $C_{\max }$

${ }^{\mathrm{a}}$ Median (range)

${ }^{\mathrm{b}}$ The analysis was on the basis of the analysis of variance model

${ }^{\mathrm{c}} N=17$

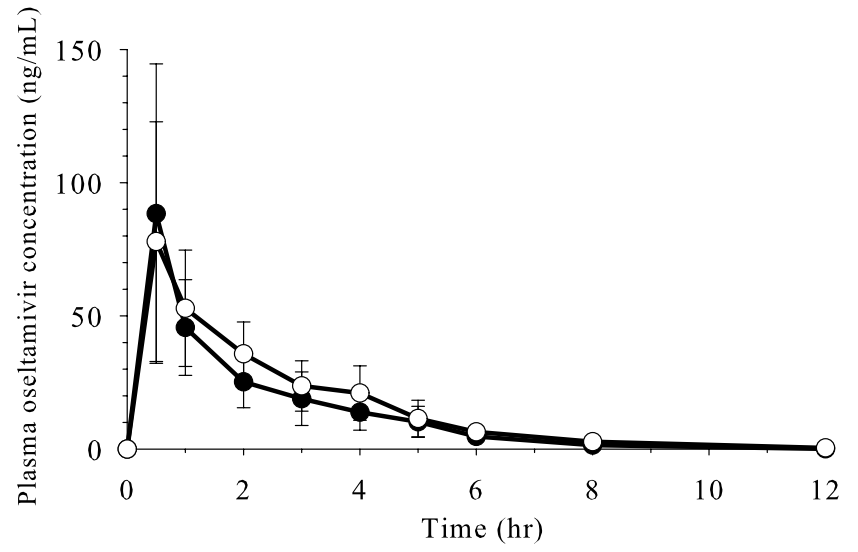

(a)

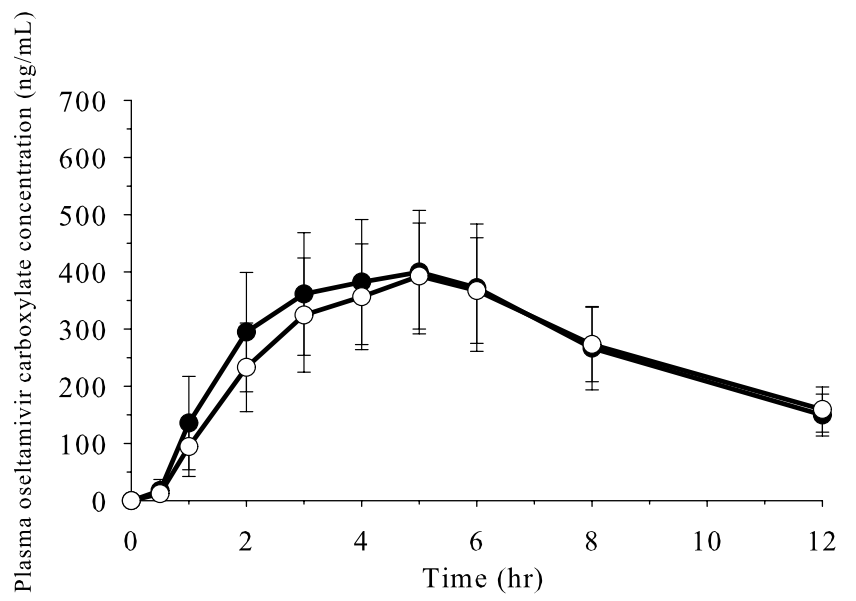

(c)

Fig. 2 Plasma concentration profiles of oseltamivir and oseltamivir carboxylate when administered with or without baloxavir marboxil. Mean ( \pm standard deviation) plasma concentration profiles of oseltamivir on day 1 (a) and day 5 (b) and those of oseltamivir car-

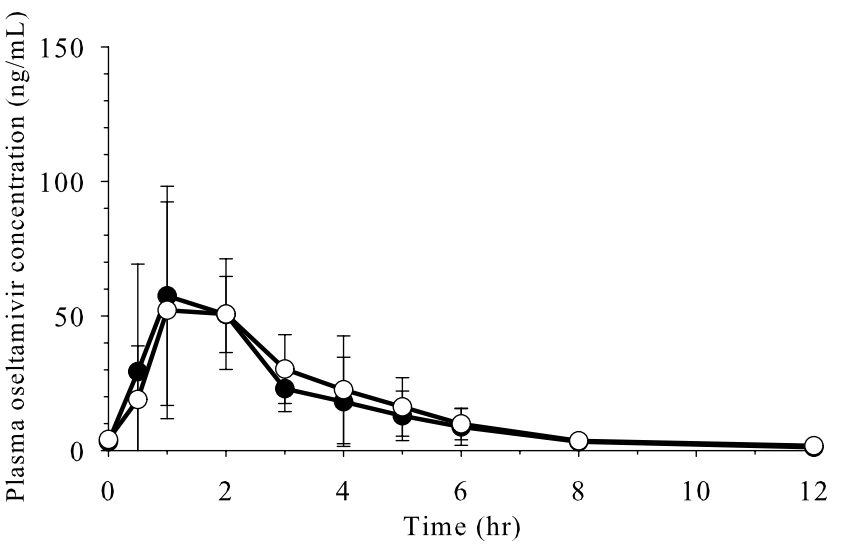

(b)

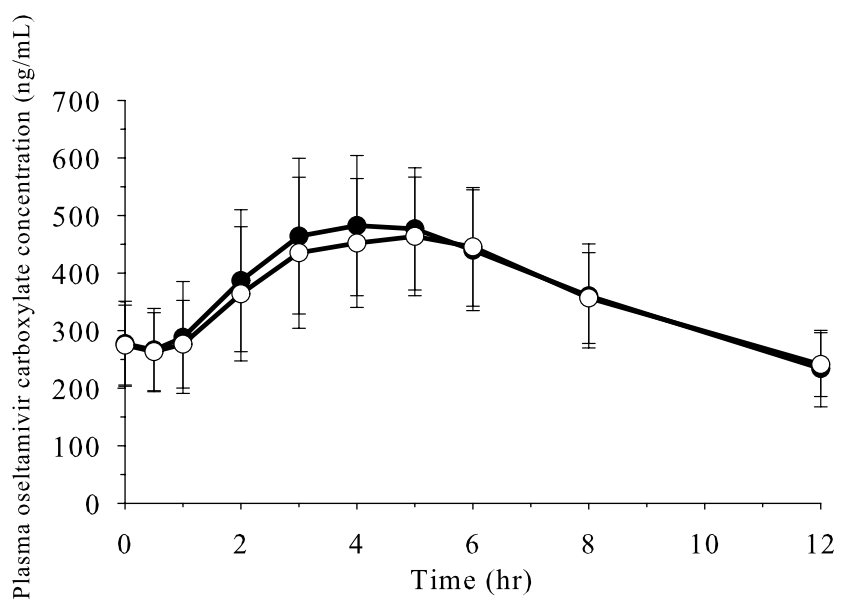

(d)

boxylate on day 1 (c) and day $5(\mathbf{d})$. A closed circle indicates treatment with oseltamivir alone ( $n=17$ on day 1 and day 5$)$ and an open circle indicates treatment with co-administration of oseltamivir and baloxavir marboxil ( $n=18$ on day $1, n=17$ on day 5 ) 
Table 2 Summary of pharmacokinetic parameters of oseltamivir when administered with or without baloxavir marboxil

\begin{tabular}{|c|c|c|c|c|c|c|}
\hline \multirow[t]{3}{*}{ Parameter } & \multicolumn{4}{|c|}{ Geometric mean (CV\%) } & \multirow{2}{*}{\multicolumn{2}{|c|}{$\begin{array}{l}\text { Geometric least-squares mean ratio (90\% confi- } \\
\text { dence interval) }\end{array}$}} \\
\hline & \multicolumn{2}{|l|}{ Oseltamivir alone } & \multicolumn{2}{|l|}{ Co-administration } & & \\
\hline & Day $1[N=17]$ & Day $5[N=17]$ & Day $1[N=18]$ & Day $5[N=17]$ & Day 1 & Day 5 \\
\hline$C_{\max }(\mathrm{ng} / \mathrm{mL})$ & $84.3(52.1)$ & $74.5(38.6)$ & $80.7(48.5)$ & $70.9(37.6)$ & $0.9664(0.8199-1.1392)$ & $0.9559(0.8263-1.1058)$ \\
\hline $\mathrm{AUC}_{0-\tau}(\mathrm{ng} \cdot \mathrm{h} / \mathrm{mL})$ & $142.2(30.9)$ & $175.5(26.0)$ & $175.6(24.6)$ & $187.2(24.0)$ & $1.2278(1.1366-1.3264)$ & $1.0672(0.9937-1.1462)$ \\
\hline$T_{\max }^{\mathrm{a}}(\mathrm{h})$ & $0.50(0.50-1.00)$ & $2.00(0.50-4.00)$ & $0.50(0.50-1.00)$ & $2.00(1.00-4.00)$ & - & - \\
\hline
\end{tabular}

$A U C_{0-\tau}$ area under the plasma concentration-time curve over the dosing interval $\tau, C_{\max }$ maximum plasma concentration, $C V \%$ coefficient of variation, $T_{\max }$ time to $C_{\max }$

${ }^{a}$ Median (range)

${ }^{\mathrm{b}}$ The analysis was on the basis of the analysis of variance model

Table 3 Summary of pharmacokinetic parameters of oseltamivir carboxylate when administered with or without baloxavir marboxil

\begin{tabular}{|c|c|c|c|c|c|c|}
\hline \multirow[t]{3}{*}{ Parameter } & \multicolumn{4}{|c|}{ Geometric mean (CV \%) } & \multirow{2}{*}{\multicolumn{2}{|c|}{$\begin{array}{l}\begin{array}{l}\text { Geometric least-squares mean ratio (90\% confi- } \\
\text { dence interval) }\end{array} \\
\text { Co-administration/oseltamivir alone }\end{array}$}} \\
\hline & \multicolumn{2}{|l|}{ Oseltamivir alone } & \multicolumn{2}{|l|}{ Co-administration } & & \\
\hline & Day $1[N=17]$ & Day $5[N=17]$ & Day $1[N=18]$ & Day $5[N=17]$ & Day 1 & Day 5 \\
\hline$C_{\max }(\mathrm{ng} / \mathrm{mL})$ & $402(29.0)$ & $493(24.2)$ & $386(22.9)$ & $474(22.5)$ & $0.9594(0.9074-1.0145)$ & $0.9648(0.9299-1.0010)$ \\
\hline $\mathrm{AUC}_{0-\tau}(\mathrm{ng} \cdot \mathrm{h} / \mathrm{mL})$ & $3063(26.3)$ & $4277(23.8)$ & $2931(23.1)$ & $4209(21.2)$ & $0.9591(0.9179-1.0021)$ & $0.9873(0.9630-1.0122)$ \\
\hline$T_{\max }^{\mathrm{a}}(\mathrm{h})$ & $5.00(3.00-6.00)$ & $4.00(3.00-6.00)$ & $5.00(4.00-6.00)$ & $5.00(3.00-8.00)$ & - & - \\
\hline
\end{tabular}

$A U C_{0-\tau}$ area under the plasma concentration-time curve over the dosing interval $\tau, C_{\max }$ maximum plasma concentration, $C V \%$ coefficient of variation, $T_{\max }$ time to $C_{\max }$

${ }^{a}$ Median (minimum, maximum)

${ }^{\mathrm{b}}$ The analysis was on the basis of the analysis of variance model

Table 4 Summary of treatment-emergent adverse events (TEAEs)

\begin{tabular}{lccc}
\hline TEAE by system organ class, preferred term & $\begin{array}{l}\text { Baloxavir marboxil alone } \\
{[N=17]}\end{array}$ & $\begin{array}{c}\text { Oseltamivir alone }[N=17] \\
\text { Co-admin- } \\
\text { istration } \\
{[N=18]}\end{array}$ \\
\hline Subjects with any TEAEs & $2(11.8)$ & $1(5.9)$ & $4(22.2)$ \\
Infections and infestations & $1(5.9)$ & 0 & $1(5.6)$ \\
Nasopharyngitis & 0 & 0 & $1(5.6)$ \\
Viral infection & $1(5.9)$ & 0 & 0 \\
Gastrointestinal disorders & 0 & 0 & $2(11.1)$ \\
Nausea & 0 & 0 & $1(11.1)$ \\
Skin and subcutaneous tissue disorders & 0 & 0 & $1(5.6)$ \\
Dermatitis contact & 0 & $1(5.9)$ & 0 \\
Investigations & $1(5.9)$ & $1(5.9)$ & 0 \\
Aspartate aminotransferase increased & $1(5.9)$ & 0 & 0 \\
Blood lactate dehydrogenase increased & $1(5.9)$ & 0 & 0 \\
\hline
\end{tabular}

Number of subjects $(\%)$

in two subjects treated with co-administration, and increased aspartate aminotransferase was reported in two subjects treated with baloxavir marboxil alone or oseltamivir alone. All treatment-emergent adverse events were mild and not 
considered to be related to the study drug. No safety concerns were reported in vital signs, clinical laboratory tests, or 12-lead electrocardiograms.

\section{Discussion}

The assessment of a DDI between baloxavir marboxil and oseltamivir was performed on the basis of plasma concentration data of each prodrug and their active forms. There was no formal calculation for the sample size. The sample size, 18 subjects in total, was selected to adequately assess the safety and pharmacokinetics of baloxavir marboxil and oseltamivir. The dose regimen of each drug was selected as the clinical doses. The approved dosage of baloxavir marboxil for adults and adolescents ( $\geq 12$ years of age) in Japan is $40 \mathrm{mg}$ for patients weighing $<80 \mathrm{~kg}$ or $80 \mathrm{mg}$ for patients weighing $\geq 80 \mathrm{~kg}$, [4] which is set to achieve similar plasma exposures to baloxavir acid. A single dose of baloxavir marboxil $40 \mathrm{mg}$ was selected regardless of body weight in this study and eventually all subjects enrolled had a body weight of less than $80 \mathrm{~kg}$. The dose regimen of oseltamivir was $75 \mathrm{mg}$ twice daily for 5 days in this study, which was the same as prescribing information for the treatment of influenza. [6].

Our non-clinical studies suggested that the combination treatment of baloxavir marboxil and oseltamivir has a synergistic antiviral activity against influenza virus. [2, 3] The combination treatment synergistically inhibited the cytopathic effect induced by influenza A/PR/8/34 in Madin-Darby canine kidney cells. [2] In mice infected with lethal doses of influenza $\mathrm{A} / \mathrm{PR} / 8 / 34$ virus, treatment with baloxavir marboxil at $0.5 \mathrm{mg} / \mathrm{kg}$ in combination with oseltamivir at 10 or $50 \mathrm{mg} / \mathrm{kg}$ (twice daily for 5 days) exerted a more potent protective effect against mortality or body weight loss compared with the treatment with each monotherapy. [3] These findings indicated that the combination treatment could be beneficial, particularly for seriously ill patients with influenza or treatment for low-sensitivity strains. The results in the current in vivo DDI study also support the combination treatment of baloxavir marboxil and oseltamivir.

\section{Conclusion}

The lack of a clinically meaningful DDI between baloxavir marboxil and oseltamivir has been established. Thus, healthcare professionals can select combination treatment of baloxavir marboxil and oseltamivir for patients with influenza without the need for dose adjustment.

\section{Compliance with Ethical Standards}

Funding This study was funded by Shionogi \& Co., Ltd.

Conflict of interest Nao Kawaguchi, Hiroki Koshimichi, Toru Ishibashi, and Toshihiro Wajima are employees of Shionogi \& Co., Ltd.

Ethics approval This study was conducted in accordance with current International Conference on Harmonisation Good Clinical Practice, all appropriate subject privacy requirements, and the ethical principles outlined in the Declaration of Helsinki. This study was conducted in accordance with all appropriate regulatory requirements and under a protocol approved by the institutional review board of the study site, Souseikai Hakata Clinic, Fukuoka, Japan.

Consent to participate Prior to enrollment, subjects received a full explanation of the nature and the objectives of the study, the safety of the drugs under investigation, and that they were free to withdraw from the study at any time without prejudice. Prior to the initiation of study-related procedures, the subjects provided written informed consent and received a copy of the signed consent form to keep as a reference.

Open Access This article is distributed under the terms of the Creative Commons Attribution-NonCommercial 4.0 International License (http://creativecommons.org/licenses/by-nc/4.0/), which permits any noncommercial use, distribution, and reproduction in any medium, provided you give appropriate credit to the original author(s) and the source, provide a link to the Creative Commons license, and indicate if changes were made.

\section{References}

1. Noshi T. Preclinical activity of baloxavir marboxil, a first-inclass endonuclease inhibitor for influenza. Shionogi \& Co., Ltd., Osaka, Japan, 2014 (Data on file).

2. Kitano M, Yamamoto A, Noshi T, Kawai M, Yoshida R, Sato A, Shishido T, Naito A. Synergistic antiviral activity of S-033188/S-033447, a novel inhibitor of influenza virus capdependent endonuclease, in combination with neuraminidase inhibitors in vitro. Abstract presented at the 27th European Congress of Clinical Microbiology and Infectious Diseases; 22-25 Apr 2017; Vienna.

3. Fukao K, Ando Y, Noshi T, Kawai M, Yoshida R, Sato A, Shishido T, Naito A. Delayed oral dosing of S-033188, a novel inhibitor of influenza virus cap-dependent endonuclease, exhibited significant reduction of viral titer and prolonged survival in mice infected with influenza A virus. Abstract presented at the 27th European Congress of Clinical Microbiology and Infectious Diseases; 22-25 Apr 2017; Vienna.

4. Xofluza [package insert] (in Japanese). Shionogi \& Co., Ltd., Osaka, Japan, 2018.

5. Koshimichi H. Safety, tolerability, and pharmacokinetics of the novel anti-influenza agent baloxavir marboxil in healthy adults: phase 1 study findings. Shionogi \& Co., Ltd., Osaka, Japan, 2015 (Data on file).

6. Tamiflu [package insert]. F. Hoffmann-La Roche Ltd., Basel, Switzerland, 1999 
7. George H, Joseph M, Penelope W. Clinical pharmacokinetics of the prodrug oseltamivir and its active metabolite Ro 64-0802. Clin Pharmacokinet. 1999;37(6):471-84.

8. Regina D, Bharat T, Enrico F, Pia S, Charles O, Penny W. Safety and pharmacology of oseltamivir in clinical use. Drug Saf. 2003;26(11):787-801.

9. Morimoto K, Nakakariya M, Shirasaka Y, Kakinuma C, Fujita T, Tamai I, Ogihara T. Oseltamivir (Tamiflu) efflux transport at the blood-brain barrier via P-glycoprotein. Drug Metab Dispos. 2008;36(1):6-9.

10. Ogihara T, Kano T, Wagatsuma T, Wada S, Yabuuchi H, Enomoto S, Morimoto K, Shirasaka Y, Kobayashi S, Tamai I. Oseltamivir (Tamiflu) is a substrate of peptide transporter 1 . Drug Metab Dispos. 2009;37(8):1676-81.
11. US Food and Drug Administration. Draft guidance: clinical drug interaction studies: study design, data analysis, and clinical implications, guidance for industry. Silver Spring, U.S.: U.S. Department of Health and Human Services Food and Drug Administration Center for Drug Evaluation and Research (CDER); 2017.

12. European Medicines Agency. Guideline on the investigation of drug interactions. London, U.K.: Committee for Human Medical Products (CHMP); 2012.

13. Ministry of Health, Labour and Welfare. Draft guideline for drugdrug interactions. Tokyo, Japan: Evaluation and Licensing Division, Pharmaceutical and Food Safety Bureau; 2017. 